\title{
Phononic Band Gap Width Control through Structural and Material Parameters in Two-Dimensional Phononic Crystals
}

\author{
I. ŚLIWA ${ }^{a}$ AND M. KRAWCZYK ${ }^{b, *}$ \\ ${ }^{a}$ Nonlinear Optics Division, Adam Mickiewicz University \\ Umultowska 85, 61-614 Poznań, Poland \\ ${ }^{b}$ Surface Physics Division, Adam Mickiewicz University \\ Umultowska 85, 61-614 Poznań, Poland \\ (Received 18 April, 2005; in final form 22 August, 2005)
}

\begin{abstract}
The phononic band structures of two-dimensional solid phononic crystals with different lattice and scatterer symmetry are studied numerically, with three types of lattice (square, triangular, and rectangular) and four different scatterer shapes (circle, hexagon, square, and rectangle) considered. $X Y$ and $Z$ vibration modes are investigated separately. Two types of phononic crystal are considered: one composed of high-density rods embedded in a low-density matrix, the other of low-density rods in a high-density matrix. In the former case, lattice type and polarization being fixed, the broadest gaps are obtained when the symmetry of the rods corresponds to that of the lattice (the shape of a rod is identical with that of the first Brillouin zone); the largest gap width values are observed in triangular lattice-based crystals (compared to those based on the square and rectangular lattices), the shape of the corresponding first Brillouin zone being closest to a circle. These rules do not apply to structures in which the density of the rod material is lower than that of the matrix. In this case, when the symmetry of the rods corresponds to that of the lattice, gaps either fail to appear at all, or are much narrower than in other configurations. The effect of other material parameter values (such as the longitudinal and transversal velocity values) on the relation between the energy gap width and the scatterer symmetry is found to be much lesser.
\end{abstract}

PACS numbers: $43.20 .+\mathrm{g}, 43.35 .+\mathrm{d}, 43.40 .+\mathrm{s}$

${ }^{*}$ corresponding author; e-mail: krawczyk@amu.edu.pl 


\section{Introduction}

Elastic wave propagation in periodic structures referred to as phononic crystals (PC) has been the subject of intensive research over the last few years [1-7]. Composed of elastic centers (e.g. cylinders, in the two-dimensional case) embedded in a matrix of a different elastic material and disposed in nodes of a crystal lattice, phononic crystals show in their band structure energy gaps forbidden to elastic wave propagation. Due to this property, these structures have extensive practical applications, e.g. in the construction of sound shields and filters [8-15], and recently also in refractive devices such as sound-wave focusing acoustic lenses [16] or acoustic interferometers $[16,17]$. Another noteworthy application of phononic crystals is their use in selective frequency waveguides [18]. In view of these applications, it is important to know how to design structures with the phononic gap as wide as possible. Beside the physical properties of the PC crystal component materials, the phononic gap width is found to depend strongly on the lattice symmetry, as well as on the scatterer shape $[19,20]$. This dependence is a common feature of phononic and photonic crystals [21]. In both crystals, the gap width depends also on the rod orientation with respect to the lattice, and thus on the relative orientation of the rods as well [20, 22].

The effect of scatterer shape and symmetry on the phononic band gap in two-dimensional solid phononic crystals has recently been studied by Kuang et al. [23]; the investigated structures consisted of steel rods embedded in epoxy, and thus represented the case of high-density rods in a low-density matrix only. Three types of lattice (triangular, hexagonal, and square) and four different scatterer shapes (hexagon, circle, square, and triangle) were considered. For a given lattice symmetry, the absolute phononic band gap width was found to be the largest when the shape and orientation of the scatterers corresponded to those of the lattice. Besides, for a given scatterer shape, the gap was found to be the broadest when the lattice had the largest coordination number, as the crystal symmetries in this case were not reduced by the scatterers. Also, the band gap was shown to be controllable by adjusting the scatterer orientation and size: the normalized gap width was found to increase if the maximum allowed filling fraction of scatterers increases after rotation; otherwise the normalized gap width would be reduced. The aim of this study is to show the effect of rod and matrix material parameters (the mass density, in particular) on the relation between the energy gap width and the symmetry of the scatterers and the crystal lattice in two-dimensional phononic crystals. This objective will be realized by investigating the dependence of the gap width on the rod symmetry, with the rods embedded in different sorts of lattice; the two cases: high-density rods in a low-density matrix, and low-density rods in a high-density matrix, will be considered separately. Four composite types will be examined first: steel/epoxy, C/epoxy, $\mathrm{Pb} /$ epoxy, and duralumin/epoxy, the epoxy, component being always the matrix. Inverse structures, i.e. epoxy/steel, epoxy/C, epoxy/Pb, and epoxy/duralumin, 
representing the case of low-density rods in a high-density matrix, will be investigated in the next step. Phononic band gaps will be studied in two-dimensional solid phononic crystals differing in lattice symmetry and rod shape. Specifically, three types of lattice: square (sq), triangular (tr), and rectangular (rc), and four different scatterer shapes: circle $(\mathrm{C})$, hexagon (HX), square (SQ), and rectangle (RC), will be considered. The rod orientation with respect to the lattice is fixed, the symmetry axes of the rods corresponding to those of the crystal lattice with the same symmetry, the configuration allowing to generate the broadest energy gaps in phononic crystals composed of two solids [23]. In our investigation of elastic wave propagation in these structures, $X Y$ modes (polarized in the wave propagation plane) and $Z$ modes (polarized in the direction perpendicular to the wave propagation) will be examined separately. The effect of rod and matrix material parameters, especially the density ratio between the rod material and the matrix material, on the relation between the energy gap width and the lattice and rod symmetry proves to be substantial for both $Z$ and $X Y$ modes.

The paper is divided into four sections. In Sect. 2, we describe the plane wave $(\mathrm{PW})$ method used in the band structure calculation; the numerical results as well as a table of material parameters are presented in Sect. 3; the results are discussed in Sect. 4.

\section{Method of calculation}

The phononic band structure is calculated through the elastic medium wave equation, solved with the use of the well-known plane wave method [4, 20, 24]. The plane wave method consists in transforming a differential wave equation, whose coefficients are periodic functions of two-dimensional position vector $\boldsymbol{r}=\left(x_{1}, x_{2}\right)$, into the reciprocal space. For a nonhomogeneous elastic medium the wave equation reads [25]

$$
\begin{gathered}
\rho(\boldsymbol{r}) \frac{\partial^{2} u_{i}}{\partial t^{2}}=\nabla \cdot\left[\rho(\boldsymbol{r}) c_{\mathrm{t}}^{2}(\boldsymbol{r}) \nabla u_{i}\right]+\nabla \cdot\left[\rho(\boldsymbol{r}) c_{\mathrm{t}}^{2}(\boldsymbol{r}) \frac{\partial \boldsymbol{u}}{\partial x_{i}}\right] \\
+\frac{\partial}{\partial x_{i}}\left[\left(\rho(\boldsymbol{r}) c_{1}^{2}(\boldsymbol{r})-2 \rho(\boldsymbol{r}) c_{\mathrm{t}}^{2}(\boldsymbol{r})\right) \nabla \cdot \boldsymbol{u}\right],
\end{gathered}
$$

where $c_{\mathrm{t}}$ and $c_{\mathrm{l}}$ are the elastic wave transversal and longitudinal velocities, respectively; $\rho$ denotes mass density, and $\boldsymbol{u}(\boldsymbol{r}, t)$ stands for the time- and space-dependent deflection vector, describing the medium molecules deflection from the balance point, and defined by its three components $u_{i}(i=1,2,3)$ in the Cartesian coordinate system $\left(0 x_{1} x_{2} x_{3}\right)$.

The structure is assumed to be homogeneous along the $x_{3}$ axis, which reduces the problem to two dimensions. If the wave propagation is confined to the phononic crystal periodicity plane (the vector $\boldsymbol{r}_{\|}=\left(x_{1}, x_{2}\right)$ plane), (1) splits into two independent equations [24]

- an equation describing $X Y$ modes, or oscillations polarized in plane $\left(x_{1} 0 x_{2}\right)$ 


$$
\frac{\partial^{2} u_{i}}{\partial t^{2}}=\frac{1}{\rho}\left\{\frac{\partial}{\partial x_{i}}\left(\lambda \frac{\partial u^{l}}{\partial x_{l}}\right)+\frac{\partial}{\partial x_{l}}\left[\mu\left(\frac{\partial u_{i}}{\partial x_{l}}+\frac{\partial u_{l}}{\partial x_{i}}\right)\right]\right\},
$$

where

$$
\begin{aligned}
& \mu=\rho c_{\mathrm{t}}^{2}, \\
& \lambda=\rho\left(c_{\mathrm{l}}^{2}-2 c_{\mathrm{t}}^{2}\right) \quad \text { and } \quad i, l=1,2
\end{aligned}
$$

- an equation describing $Z$ modes, or oscillations polarized along the rod axis, i.e. along vector $\boldsymbol{e}_{3}\left(\boldsymbol{e}_{3} \perp \boldsymbol{k}\right)$

$$
\rho\left(\boldsymbol{r}_{\|}\right) \frac{\partial^{2} u_{3}}{\partial t^{2}}=\nabla \cdot\left[\mu \nabla u_{3}\right]
$$

As the coefficients in equations of motion (2) and (3) are periodic functions, through Bloch's theorem, the deflection vector, $\boldsymbol{u}(\boldsymbol{r}, t)$, can be expressed as [20]

$$
\boldsymbol{u}\left(\boldsymbol{r}_{\|}, t\right)=\exp \left(\mathrm{i}\left(\boldsymbol{k} \cdot \boldsymbol{r}_{\|}-\omega t\right)\right) \sum_{\boldsymbol{G}} \boldsymbol{u}_{\boldsymbol{k}}(\boldsymbol{G}) \exp \left(\mathrm{i} \boldsymbol{G} \cdot \boldsymbol{r}_{\|}\right)
$$

$\boldsymbol{k}$ being a two-dimensional Bloch vector, and $\boldsymbol{G}$ denoting a two-dimensional reciprocal lattice vector.

Those of the material parameters which are periodic functions can be Fourier-expanded. For $Z$ modes, described by (3), Fourier expansion can be applied to the inverse of mass density, $\rho^{-1}$, and to coefficient $\mu$; in (2), describing $X Y$ modes, the Fourier-expandable parameters are $\rho^{-1}, \mu$, and $\lambda$

$$
\begin{aligned}
& \rho^{-1}\left(\boldsymbol{r}_{\|}\right)=\sum_{\boldsymbol{G}} \rho^{-1}(\boldsymbol{G}) \exp \left(\mathrm{i} \boldsymbol{G} \cdot \boldsymbol{r}_{\|}\right), \\
& \mu\left(\boldsymbol{r}_{\|}\right)=\sum_{\boldsymbol{G}} \mu(\boldsymbol{G}) \exp \left(\mathrm{i} \boldsymbol{G} \cdot \boldsymbol{r}_{\|}\right) \\
& \lambda\left(\boldsymbol{r}_{\|}\right)=\sum_{\boldsymbol{G}} \lambda(\boldsymbol{G}) \exp \left(\mathrm{i} \boldsymbol{G} \cdot \boldsymbol{r}_{\|}\right) .
\end{aligned}
$$

Coefficients $\rho^{-1}(\boldsymbol{G}), \mu(\boldsymbol{G})$, and $\lambda(\boldsymbol{G})$ in the above expansions are calculated from the inverse Fourier transformation. All these coefficients will have the same form; for example, the inverse of mass density will read

$$
\rho^{-1}(\boldsymbol{G})=\frac{1}{S} \iint \rho^{-1}\left(\boldsymbol{r}_{\|}\right) \exp \left(-\mathrm{i} \boldsymbol{G} \cdot \boldsymbol{r}_{\|}\right) \mathrm{d} \boldsymbol{r}_{\|},
$$

the integration covering the two-dimensional unit cell surface, $S$. When $\boldsymbol{G}=0$, Eq. (8) defines the mean value

$$
\rho^{-1}(\boldsymbol{G}=0)=\bar{\rho}^{-1} \equiv \rho_{\mathrm{A}}^{-1} f+\rho_{\mathrm{B}}^{-1}(1-f),
$$

where $\rho_{\mathrm{A}}$ is the mass density value in the $\operatorname{rod}$ (material A), $\rho_{\mathrm{B}}$ is its matrix counterpart (material B), and $f=S_{\mathrm{r}} / S$ denotes the filling fraction $\left(S_{\mathrm{r}}\right.$ being the rod cross-section area). When $\boldsymbol{G} \neq 0$, Eq. (8) becomes

$$
\rho^{-1}(\boldsymbol{G} \neq 0)=\left(\rho_{\mathrm{A}}^{-1}-\rho_{\mathrm{B}}^{-1}\right) I(\boldsymbol{G})=\Delta \rho^{-1} I(\boldsymbol{G}),
$$

where $\Delta \rho^{-1}=\rho_{\mathrm{A}}^{-1}-\rho_{\mathrm{B}}^{-1}$, and $I(\boldsymbol{G})$ is a structural factor determined by the rod shape 


$$
I(\boldsymbol{G}) \equiv \frac{1}{S} \iint_{S_{\mathrm{r}}} \exp \left(-\mathrm{i} \boldsymbol{G} \cdot \boldsymbol{r}_{\|}\right) \mathrm{d} \boldsymbol{r}_{\|}
$$

The integration in (11) is performed over the rod cross-section. For the four types of rods (Fig. 1), with circular, hexagonal, rectangular, or square cross-section, the integration results in the formulae $I_{\mathrm{C}}(\boldsymbol{G}), I_{\mathrm{HX}}(\boldsymbol{G}), I_{\mathrm{RC}}(\boldsymbol{G})$, and $I_{\mathrm{SQ}}(\boldsymbol{G})$ denoting the respective structural factors are presented in the appendix.

a)

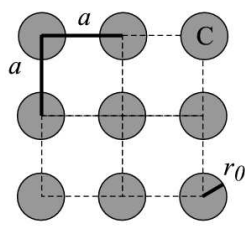

e)

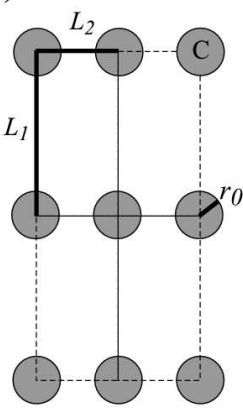

i)

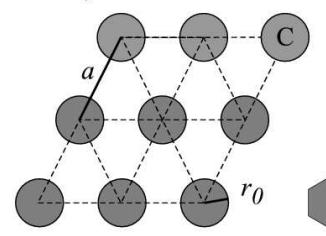

b)

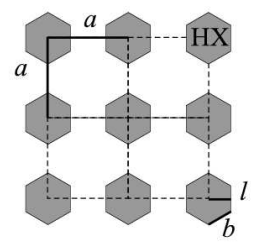

f)

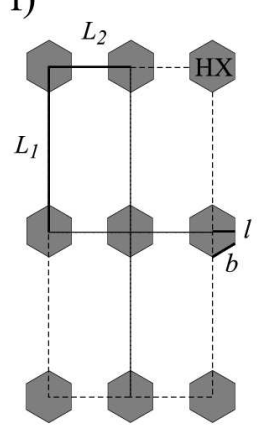

j) c)

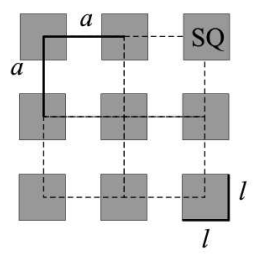

g)

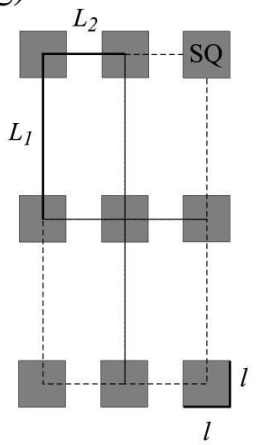

k) d)

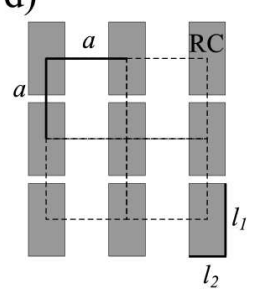

h)

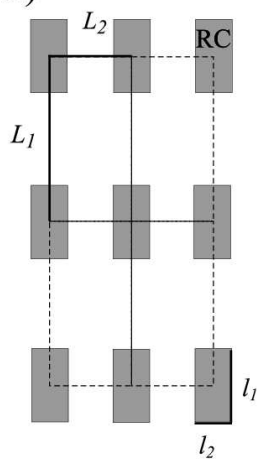

1)
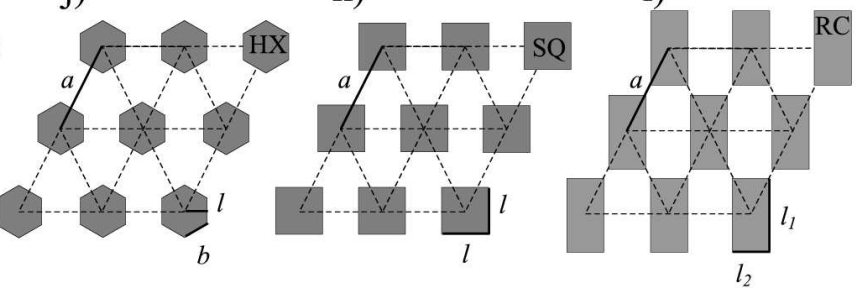

Fig. 1. Models of two-dimensional phononic crystals based on different types of lattice and with different rod cross-section shapes: square (sq) lattice-based crystals (the lattice constant being $a$ ) with rod cross-section in the shape of: circle $\mathrm{C}$ with radius $r_{0}$ (a), regular hexagon HX with side length $b(\mathrm{~b})$, square SQ with side length $l(\mathrm{c})$, and rectangle $\mathrm{RC}$ with side lengths $l_{1}$ and $l_{2}(\mathrm{~d})$; rectangular (rc) lattice-based crystals (the lattice constants being $L_{1}$ and $L_{2}$ with rod cross-section in the shape of: circle (e), hexagon (f), square $(\mathrm{g})$, and rectangle (h); triangular (tr) lattice-based crystals (the lattice constant being $a$ ) with rod cross-section in the shape of: circle (i), hexagon (j), square (k), and rectangle (l). 
The substitution of (4), (5-7), (9), and (10) into (2) leads to an infinite system of algebraic equations for $X Y$ mode eigenvalues $\omega(\boldsymbol{k})$

$$
\begin{aligned}
& \omega^{2} u_{\boldsymbol{k}}^{i}(\boldsymbol{G})=\sum_{\boldsymbol{G}}\left\{\sum _ { l , \boldsymbol { G } ^ { \prime \prime } } \rho ^ { - 1 } ( \boldsymbol { G } - \boldsymbol { G } ^ { \prime \prime } ) \left[\lambda\left(\boldsymbol{G}^{\prime \prime}-\boldsymbol{G}^{\prime}\right)\left(\boldsymbol{k}+\boldsymbol{G}^{\prime \prime}\right)_{l}\left(\boldsymbol{k}+\boldsymbol{G}^{\prime \prime}\right)_{i}\right.\right. \\
& \left.+\mu\left(\boldsymbol{G}^{\prime \prime}-\boldsymbol{G}^{\prime}\right)\left(\boldsymbol{k}+\boldsymbol{G}^{\prime}\right)_{i}\left(\boldsymbol{k}+\boldsymbol{G}^{\prime \prime}\right)_{l}\right] u_{\boldsymbol{k}}^{l}\left(\boldsymbol{G}^{\prime}\right) \\
& \left.+\sum_{\boldsymbol{G}^{\prime \prime}}\left[\rho^{-1}\left(\boldsymbol{G}-\boldsymbol{G}^{\prime \prime}\right) \mu\left(\boldsymbol{G}^{\prime \prime}-\boldsymbol{G}^{\prime}\right) \sum_{j}\left(\boldsymbol{k}+\boldsymbol{G}^{\prime}\right)_{j}\left(\boldsymbol{k}+\boldsymbol{G}^{\prime \prime}\right)_{j}\right] u_{\boldsymbol{k}}^{i}\left(\boldsymbol{G}^{\prime}\right)\right\},
\end{aligned}
$$

where $i, j, l=1,2$. Analogically, the following $Z$ mode eigenproblem is deduced from $(3)$

$$
\omega^{2} u_{\boldsymbol{k}}^{3}(\boldsymbol{G})=\sum_{\boldsymbol{G}^{\prime}, \boldsymbol{G}^{\prime \prime}} \rho^{-1}\left(\boldsymbol{G}-\boldsymbol{G}^{\prime \prime}\right) \mu\left(\boldsymbol{G}^{\prime \prime}-\boldsymbol{G}^{\prime}\right)\left(\boldsymbol{k}+\boldsymbol{G}^{\prime}\right) \cdot\left(\boldsymbol{k}+\boldsymbol{G}^{\prime \prime}\right) u_{\boldsymbol{k}}^{3}\left(\boldsymbol{G}^{\prime}\right)
$$

\section{Numerical results and discussion}

The material parameter values $\left(\rho, c_{\mathrm{l}}, c_{\mathrm{t}}\right)$ in all the materials involved (C, epoxy, steel, $\mathrm{Pb}$, and duralumin), as well as the rod/matrix parameter value contrasts $\left(\Delta \rho=\left|\rho_{\mathrm{A}}-\rho_{\mathrm{B}}\right|, \Delta c_{\mathrm{l}}=\left|c_{\mathrm{l}}^{\mathrm{A}}-c_{\mathrm{l}}^{\mathrm{B}}\right|, \Delta c_{\mathrm{t}}=\left|c_{\mathrm{t}}^{\mathrm{A}}-c_{\mathrm{t}}^{\mathrm{B}}\right|\right)$, are specified in Table I.

TABLE I

Mass density, transversal velocity, and longitudinal velocity values in the component materials (C [20], epoxy [20], steel [26], $\mathrm{Pb}[26]$, duralumin [27]) of the studied phononic crystals. Parameter value contrasts between the rods and the matrix $(\Delta \rho$, $\Delta c_{1}$ and $\left.\Delta c_{\mathrm{t}}\right)$ are specified as well.

\begin{tabular}{c|c|c|c|c|c|c}
\hline \hline Material & $\begin{array}{c}\rho \\
{\left[\mathrm{kg} / \mathrm{m}^{3}\right]}\end{array}$ & $\begin{array}{c}\Delta \rho \\
{\left[\mathrm{kg} / \mathrm{m}^{3}\right]}\end{array}$ & $\begin{array}{c}c_{\mathrm{t}} \\
{[\mathrm{m} / \mathrm{s}]}\end{array}$ & $\begin{array}{c}\Delta c_{\mathrm{t}} \\
{[\mathrm{m} / \mathrm{s}]}\end{array}$ & $\begin{array}{c}c_{\mathrm{l}} \\
{[\mathrm{m} / \mathrm{s}]}\end{array}$ & $\begin{array}{c}\Delta c_{\mathrm{l}} \\
{[\mathrm{m} / \mathrm{s}]}\end{array}$ \\
\hline $\mathrm{C}$ & 1750 & & 7100 & & 13310 & \\
epoxy & 1200 & 550 & 1160 & 5940 & 2830 & 10480 \\
\hline steel & 7800 & & 3220 & & 5940 & \\
epoxy & 1200 & 6600 & 1160 & 2060 & 2830 & 3110 \\
\hline $\mathrm{Pb}$ & 11357 & & 860 & & 2158 & \\
epoxy & 1200 & 10157 & 1160 & 300 & 2830 & 672 \\
\hline duralumin & 2799 & & 3095 & & 6342 & \\
epoxy & 1200 & 1599 & 1160 & 1935 & 2830 & 3512
\end{tabular}

The material parameter value contrasts vary substantially with structure composition, $\Delta \rho$ ranging from 550 to $10157, \Delta c_{\mathrm{t}}$ from 300 to 5940 , and $\Delta c_{1}$ from 672 to 10480 . This variety increases the generality of our conclusions, drawn from 
the results obtained for different phononic crystals, and regarding the effect of the rod and lattice symmetry on the energy gap width in their elastic wave spectrum.

In the numerical solution of $(12)$ and $(13), I(\boldsymbol{G})$ is substituted by $I_{\mathrm{C}}(\boldsymbol{G})$ for cylindrical rods $(17), I_{\mathrm{HX}}(\boldsymbol{G})$ for hexagonal rods $(18), I_{\mathrm{RC}}(\boldsymbol{G})$ for rectangular rods $(19)$ and $I_{\mathrm{SQ}}(\boldsymbol{G})$ for square ones (20). The number of reciprocal lattice vectors involved in Fourier expansions $(5-7)$ is limited by the condition $-N \leq n_{1}, n_{2} \leq+N$, confining the reciprocal lattice vector component values $n_{1}$ and $n_{2}$ to interval $[-N,+N]$. From the resulting finite system of algebraic equations for $6 N+3$ coefficients $u_{\boldsymbol{k}}^{i}(\boldsymbol{G}), 6 N+3$ allowed frequencies $\omega(\boldsymbol{k})$ are deduced for each wave vector from the first Brillouin zone. All the results presented in this paper were obtained for $N=7$. Some dispersion relations were calculated for $N=10$ as well, and found to be in a very good conformity with those obtained for $N=7$, regardless of the rod shape.

Among the rod shapes considered, only the rectangular cross-section leaves some arbitrariness, which lies in the rectangle side ratio, $l_{1} / l_{2}$. In order to find the optimum side ratio, i.e. that corresponding to the broadest energy gap, in a rectangular lattice-based crystal with rectangular rods, the dependence of the gap width on the filling fraction was investigated for the following values of $l_{1} / l_{2}$ : $1 / 2,1,2$ and 3 , the lattice constant ratio being fixed at $L_{1} / L_{2}=2$. Some of the results obtained for the steel/epoxy crystal are shown in Table II. Energy gap

\section{TABLE II}

The broadest energy gap width values in steel/epoxy crystals consisting of rectangular rods of different $l_{1} / l_{2}$ values disposed in nodes of the rectangular lattice $\left(L_{1} / L_{2}=2\right)$.

\begin{tabular}{c|c|c}
\hline \hline$l_{1} / l_{2}$ & $Z$ mode & $X Y$ mode \\
\hline $1 / 2$ & 0 & 0 \\
1 & 0 & 0 \\
2 & 0.66 & 0.50 \\
3 & 0.47 & 0.22
\end{tabular}

width values, as well as frequency values, are specified, throughout this study, in the units of reduced frequency, $\Omega$, defined as follows:

$$
\Omega=\frac{\omega a_{0}}{4 \pi \sqrt{\bar{c}_{\mathrm{t}}}}
$$

$a_{0}$ being the distance between two neighboring lattice nodes, and $\bar{c}_{\mathrm{t}}$ denoting the mean between the transversal velocities in materials $\mathrm{A}$ and $\mathrm{B}$. The energy gap is found to be the broadest for the side ratio equal to the constant lattice ratio, $l_{1} / l_{2}=2$ in the case considered. Therefore, we have confined the scope of our analysis to rectangular rods with a side ratio $l_{1} / l_{2}=2$ and a rectangular lattice with the constant lattice ratio $L_{1} / L_{2}=2$. The specific value of the constant 
lattice ratio, as well as that of the side length ratio, is of no importance for this study as long as both ratios are equal, which means that the symmetry of the rods corresponds to that of the lattice. In order to find the optimum filling fraction value, i.e. that corresponding to the maximum width of the broadest gap - the reduced frequency was plotted against the filling fraction, $f$, on the basis of (12) and (13), with three possible lattice types (square, triangular, and rectangular), four rod cross-section shapes (square, circle, regular hexagon, and rectangle) and two polarizations considered in each material composition.
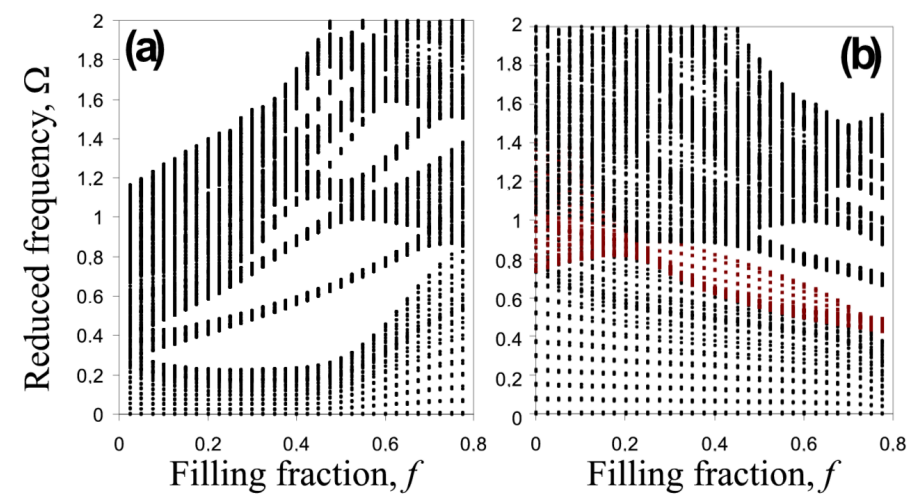

Fig. 2. $Z$ mode reduced frequency plotted against the filling fraction, $f$, for a steel/epoxy crystal with cylindrical rods disposed in square lattice nodes (a), and its epoxy/steel counterpart (b).

Figure 2 shows some examples of phononic crystal energy bands plotted against the filling fraction in two cases: (a) in a steel/epoxy crystal, representing the case of high-density rods in a low-density matrix $\left(\rho_{\mathrm{B}}<\rho_{\mathrm{A}}\right)$, and (b) in an epoxy/steel structure, with low-density rods in a high-density matrix $\left(\rho_{\mathrm{B}}>\rho_{\mathrm{A}}\right)$. These two qualitatively different cases bring out the significant effect of the material parameter values on the relations obtained. In the case of high-density rods in a low-density matrix, the optimum filling fraction value for the broadest gap (found between the first and the second band) is $f=0.48$, the gap center shifting up the frequency scale as $f$ increases (Fig. 2a). In the other case, the gaps are narrower, their centers shift down as $f$ increases, and the optimum filling fraction value is much higher than in the case considered previously (Fig. 2b). Therefore, in the following sections of this paper, the cases of high-density rods in a low-density matrix and low-density rods in a high-density matrix will be considered separately.

\subsection{The case of high-density rods in a low-density matrix}

In this paragraph we shall focus on structures in which the matrix material is assumed to be epoxy; due to the lowest mass density of this material, inequality $\rho_{\mathrm{A}}>\rho_{\mathrm{B}}$, conducive to the appearance of wider energy gaps [24], is fulfilled in each case. The width values of the broadest energy gaps obtained in each of the 
examined structures, and corresponding to the optimum filling fraction values, are specified in Table III.

\section{TABLE III}

The width values of the broadest energy gap in the examined phononic crystals, in reduced frequency units. sq denotes square lattice, $\operatorname{tr}$ - triangular lattice, $\mathrm{rc}$ - rectangular lattice, $\mathrm{C}$ - cylindrical rods, HX - hexagonal rods, SQ square rods, and $\mathrm{RC}$ - rectangular rods.

\begin{tabular}{|c|c|c|c|c|c|c|}
\hline Material & Polarization & Lattice & $\mathrm{C}$ & HX & SQ & $\mathrm{RC}$ \\
\hline \multirow{6}{*}{$\begin{array}{l}\text { steel } \\
\text { /epoxy }\end{array}$} & \multirow{3}{*}{$Z$} & $\mathrm{sq}$ & 0.37 & 0.36 & 0.64 & 0.20 \\
\hline & & $\operatorname{tr}$ & 0.77 & 0.80 & 0.75 & 0.31 \\
\hline & & $\mathrm{rc}$ & 0.04 & 0.05 & 0 & 0.47 \\
\hline & \multirow{3}{*}{$X Y$} & $\mathrm{sq}$ & 0.57 & 0.51 & 0.74 & 0.06 \\
\hline & & $\operatorname{tr}$ & 0.86 & 0.86 & 0.75 & 0.11 \\
\hline & & $\mathrm{rc}$ & 0.03 & 0 & 0 & 0.35 \\
\hline \multirow{6}{*}{ C/epoxy } & \multirow{3}{*}{$Z$} & $\mathrm{sq}$ & 0.21 & 0.16 & 0.30 & 0.02 \\
\hline & & $\operatorname{tr}$ & 0.49 & 0.55 & 0.48 & 0.11 \\
\hline & & $\mathrm{rc}$ & 0 & 0.16 & 0 & 0.27 \\
\hline & \multirow{3}{*}{$X Y$} & sq & 0.13 & 0.11 & 0.37 & 0 \\
\hline & & $\operatorname{tr}$ & 0.47 & 0.49 & 0.40 & 0 \\
\hline & & $\mathrm{rc}$ & 0 & 0 & 0 & 0.09 \\
\hline \multirow{6}{*}{$\mathrm{Pb} /$ epoxy } & \multirow{3}{*}{$Z$} & $\mathrm{sq}$ & 0.35 & 0.35 & 0.35 & 0.24 \\
\hline & & $\operatorname{tr}$ & 0.47 & 0.47 & 0.46 & 0.18 \\
\hline & & $\mathrm{rc}$ & 0.08 & 0 & 0.09 & 0.18 \\
\hline & \multirow{3}{*}{$X Y$} & $\mathrm{sq}$ & 0.32 & 0.32 & 0.32 & 0.16 \\
\hline & & $\operatorname{tr}$ & 0.37 & 0.37 & 0.36 & 0.27 \\
\hline & & $\mathrm{rc}$ & 0 & 0 & 0 & 0.07 \\
\hline \multirow{6}{*}{$\begin{array}{l}\text { duralumin } \\
\text { /epoxy }\end{array}$} & \multirow{3}{*}{$Z$} & $\mathrm{sq}$ & 0.21 & 0.20 & 0.35 & 0.07 \\
\hline & & $\operatorname{tr}$ & 0.48 & 0.51 & 0.44 & 0.21 \\
\hline & & $\mathrm{rc}$ & 0 & 0 & 0 & 0.18 \\
\hline & \multirow{3}{*}{$X Y$} & $\mathrm{sq}$ & 0.29 & 0.25 & 0.37 & 0 \\
\hline & & $\operatorname{tr}$ & 0.47 & 0.47 & 0.40 & 0.06 \\
\hline & & $\mathrm{rc}$ & 0 & 0 & 0 & 0.08 \\
\hline
\end{tabular}

The results shown in Table III indicate that, among the lattice types considered here, it is the triangular lattice (i.e. the one whose first Brillouin zone has a shape closest to a circle) that allows one to obtain energy gaps which are by far the broadest. Besides, the gaps obtained in each of the phononic crystals covered by Table III are found to be the broadest in structures in which the symmetry of the rods corresponds exactly to that of the lattice, which confirms the results reported 
in [23]. The results obtained in the case of triangular lattice can be expressed by the following inequality, for both $Z$ and $X Y$ modes:

$\Delta \Omega_{\mathrm{HX}} \geq \Delta \Omega_{\mathrm{C}} \geq \Delta \Omega_{\mathrm{SQ}} \geq \Delta \Omega_{\mathrm{RC}}$,

where $\Delta \Omega_{\mathrm{SQ}}$ is the gap width in the square-rod composite, $\Delta \Omega_{\mathrm{C}}$ is the corresponding value in the cylinder-rod structure, $\Delta \Omega_{\mathrm{HX}}$ denotes the gap width in the composite with hexagonal rods and $\Delta \Omega_{\mathrm{RC}}$ refers to the case of rectangular rods.

The results obtained in the case of square lattice, for both $Z$ and $X Y$ modes, can be expressed as follows:

$\Delta \Omega_{\mathrm{SQ}} \geq \Delta \Omega_{\mathrm{C}} \geq \Delta \Omega_{\mathrm{HX}}>\Delta \Omega_{\mathrm{RC}}$.

In the case of rectangular lattice, the results obtained cannot be expressed by just one inequality; however, the broadest gaps are found to appear for rectangular rods.

The results obtained for steel/epoxy, C/epoxy, and duralumin/epoxy structures are qualitatively the same; in the case of $Z$ modes, all the weak inequalities in (15) and (16) can be replaced with strict ones. However, the results obtained for $\mathrm{Pb}$ /epoxy are different: as long as the lattice type is fixed, gaps appearing at various rod shapes (cylindrical, hexagonal, and square) are found to be equal. The optimum filling fraction value lies within the range from 0.460 to 0.675 in each of the first three cases, but is found to be below $f=0.375$, and thus relatively low, in the case of the $\mathrm{Pb} /$ epoxy composition; this means that the matrix material is then the dominant one in the $\mathrm{Pb} /$ epoxy crystal. The described properties of the energy gap width in the $\mathrm{Pb}$ /epoxy crystal are due to different contrasts of longitudinal and transversal velocity values in the component materials: the relations between the $c_{1}$ and $c_{\mathrm{t}}$ values in the rods and in the matrix are reversed with respect to the other compositions $\left(c_{\mathrm{tA}}<c_{\mathrm{tB}}\right.$ and $\left.c_{\mathrm{lA}}<c_{\mathrm{BB}}\right)$, while the density relation is the same $\left(\rho_{\mathrm{A}}>\rho_{\mathrm{B}}\right)$. The gaps are found to be narrowest in the $\mathrm{C} /$ epoxy composite, due to the lowest density contrast, $\Delta \rho=550$, between the component materials.

\subsection{The case of low-density rods in a high-density matrix}

In order to check whether the results discussed above depend on the rod and matrix density values, similar computations of the phononic gap width values for different rod symmetries were performed for epoxy/steel, epoxy/C, epoxy/Pb, and epoxy/duralumin crystals, representing the case of low-density rods in a high-density matrix. Providing an example, Fig. 2 b shows the reduced frequency plotted against the filling fraction on the basis of the results obtained in the epoxy/steel crystal with cylindrical rods embedded in a square lattice.

In Fig. 3 the gap width is plotted against the rod shape in steel/epoxy and epoxy/steel crystals (Fig. $3 \mathrm{a}$ and b) as well as in $\mathrm{Pb} /$ epoxy and epoxy/Pb crystals (Fig. 3c and d); $Z$ and $X Y$ modes are considered separately (Fig. 3a, c, and b, d, respectively). The plots obtained for the remaining two compositions, i.e. for $\mathrm{C} /$ epoxy and duralumin/epoxy, are qualitatively the same as those obtained for the steel/epoxy crystal, and thus are not depicted in the figures presented in this 

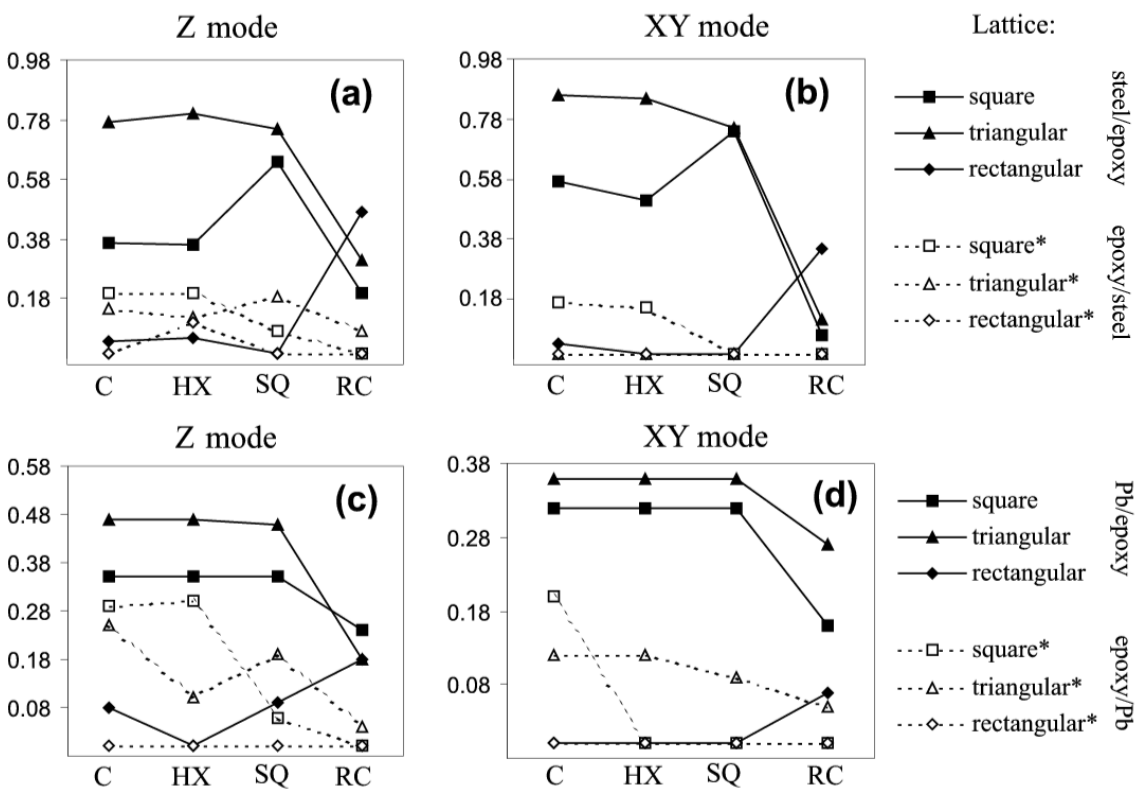

Fig. 3. The energy gap width (in the units of reduced frequency $\Omega$ ) plotted against the rod shape (C, HX, SQ, RC) in two-dimensional phononic crystals; (a) and (b) show results obtained in steel/epoxy and epoxy/steel crystals; (c) and (d) present those obtained for $\mathrm{Pb} /$ epoxy and epoxy/Pb compositions. $Z$ and $X Y$ modes are considered separately, the results being depicted in (a), (c), and (b), (d), respectively. C denotes cylindrical rods, HX - hexagonal rods, SQ - square rods, and RC - rectangular rods; the rods are disposed in nodes of a square, a triangular or a rectangular lattice, as indicated by symbols explained in the legend. The asterisk in the legend refers to the epoxy/steel structures in (a) and (b), and to the epoxy/Pb structures in (c) and (d).

paper. The plots shown in Fig. 3 indicate clearly that, regardless of polarization, relations $(15,16)$, obtained for crystals with high-density rods in a low-density matrix (represented by the solid lines), do not apply to the inverse structures (dashed lines), in which the density of the rod material is lower than that of the matrix. In triangular lattice-based crystals, hexagonal rods are found to generate narrower gaps than cylindrical or square rods. In square lattice-based structures, square rods are found to generate no gaps at all (though other rod shapes do generate them); also in rectangular lattice-based structures no gaps are generated by rectangular rods. Therefore, the inversion of the material composition clearly implies a radical change in the relations between the gap width and the scatterer symmetry: in none of the cases considered, for a given lattice type, the broadest gaps are found to appear when the symmetry of the rods corresponds to that of the lattice. Besides, the gaps obtained in such inverse structures are much narrower (four times in the epoxy/steel crystal, and twice in the epoxy/Pb structure) than 
those observed in the corresponding crystal with high-density rods in a low-density matrix. As similar results were obtained for all the compositions considered (also in the epoxy $/ \mathrm{Pb}$ crystal with different longitudinal and transversal velocity contrasts), the strongest impact on the considered relations can be expected to come from the mass density.

\section{Conclusion}

As indicated by the results presented in the preceding section, covering two classes of phononic crystals: those with high-density rods embedded in a low-density matrix, and those with low-density rods in a high-density matrix, material-parameter values have much of an impact on the relation between the energy gap width and the lattice and rod symmetry; the effect of the mass density was found to be especially significant. In structures with high-density rods in a lowdensity matrix, the gaps are found to be the broadest when the symmetry of the rods corresponds to that of the lattice, i.e. for hexagonal rods in triangular lattice-based crystals, square rods in square lattice-based crystals, and rectangular rods in rectangular lattice-based crystals, as already demonstrated in [23]. However, this rule will cease to apply when the density of the rod material becomes lower than that of the matrix. In this case, when the symmetry of the rods corresponds to that of the lattice, gaps either fail to appear at all, or are much narrower than in other configurations. Gaps obtained in such inverse structures are always much narrower than those appearing in crystals with high-density rods in a low-density matrix. This is due to the fact that low-density rods embedded in a high-density matrix are weak scattering centers: their wave scattering effect is hardly noticeable and much lesser than that produced by high-density rods in a low-density matrix. As regards the longitudinal and transversal velocity values $\left(c_{1}\right.$ and $\left.c_{\mathrm{t}}\right)$ in the component materials, the effect of these parameters on the investigated relations proves to be less significant.

\section{Acknowledgments}

The authors are indebted to prof. H. Puszkarski for suggesting this study and for stimulating discussions. This study was supported in part by the State Committee for Scientific Research (Poland), grants KBN-2P03B 12023 and PBZKBN-044/P03-2001 (M.K.) also thanks Poznan Supercomputing and Networking Center for the access to the supercomputers, on which calculations were performed.

\section{Appendix}

Structural factors for different rod shapes:

- for cylindrical rods [4]:

$$
I_{\mathrm{C}}(\boldsymbol{G})=2 f \frac{J_{1}\left(G r_{0}\right)}{G r_{0}},
$$

where $J_{1}$ is a first-order Bessel function, $r_{0}$ is the cylinder cross-section radius, and filling fraction $f$ is defined as follows: 


$$
\begin{aligned}
& f=\frac{\pi r_{0}^{2}}{a^{2}}, \quad 0 \leq f \leq \frac{\pi}{4} \quad \text { for a square lattice, } \\
& f=\frac{4 \pi r_{0}^{2}}{a^{2} \sqrt{3}}, \quad 0 \leq f \leq \frac{\pi}{\sqrt{3}} \quad \text { for a triangular lattice, }
\end{aligned}
$$

and

$$
f=\frac{\pi r_{0}^{2}}{L_{1} L_{2}} \quad \text { for a rectangular lattice; }
$$

- for hexagonal rods [23]:

$$
\begin{gathered}
I_{\mathrm{HX}}(\boldsymbol{G})= \\
\left\{\begin{array}{c}
\frac{2 f}{3 G_{1} l}\left[\sin \left(G_{1} l\right)+\frac{1-\cos \left(G_{1} l\right)}{G_{1} l}\right], \quad G_{1} \neq 0, \quad G_{2}=0, \\
\frac{f}{3 G_{1} l}\left[\frac{1-\cos \left(2 G_{1} l\right)}{2 G_{1} l}+\sin \left(2 G_{1} l\right)\right], \quad\left|G_{1}\right|=\left|G_{2}\right| / \sqrt{3}, \quad G_{2} \neq 0, \\
\frac{f}{G_{2} l}\left[\frac{\cos \left(G_{2} l / \sqrt{3}-G_{1} l\right)-\cos \left(2 G_{2} l / \sqrt{3}\right)}{\left(G_{2}+G_{1} \sqrt{3}\right) l}+\frac{\cos \left(G_{2} l / \sqrt{3}+G_{1} l\right)-\cos \left(2 G_{2} l / \sqrt{3}\right)}{\left(G_{2}-G_{1} \sqrt{3}\right) l}\right], \\
G_{1}|\neq| G_{2}|/ \sqrt{3}, \quad| G_{2} \mid \neq 0,
\end{array}\right.
\end{gathered}
$$

where $l=b \sqrt{3} / 2, b$ denoting the regular hexagon side length; the corresponding filling fraction is:

$$
\begin{aligned}
& f=\frac{3 b^{2} \sqrt{3}}{2 a^{2}}, \quad 0 \leq f \leq \frac{3 \sqrt{3}}{8} \text { for a square lattice, } \\
& f=\frac{3 b^{2}}{a^{2}}, \quad 0 \leq f \leq 1 \quad \text { for a triangular lattice, }
\end{aligned}
$$

and

$$
f=\frac{3 b^{2} \sqrt{3}}{2 L_{1} L_{2}} \quad \text { for a rectangular lattice; }
$$

- for rectangular rods:

$$
I_{\mathrm{RC}}(\boldsymbol{G})=\left\{\begin{array}{lll}
\frac{2 l_{1}}{G_{2}} \sin \left(\frac{l_{2} G_{2}}{2}\right), & G_{1}=0, & G_{2} \neq 0, \\
\frac{2 l_{2}}{G_{1}} \sin \left(\frac{l_{1} G_{1}}{2}\right), & G_{2}=0, & G_{1} \neq 0, \\
\frac{4}{G_{1} G_{2}} \sin \left(\frac{l_{1} G_{1}}{2}\right) \sin \left(\frac{l_{2} G_{2}}{2}\right), & G_{1} \neq 0, \quad G_{2} \neq 0,
\end{array}\right.
$$

where $l_{1}$ and $l_{2}$ are the rectangle side lengths, and filling fraction $f$ reads

$$
\begin{aligned}
& f=\frac{l_{1} l_{2}}{a^{2}} \quad \text { for a square lattice, } \\
& f=\frac{2 l_{1} l_{2}}{a^{2} \sqrt{3}} \quad \text { for a triangular lattice, }
\end{aligned}
$$

and

$$
f=\frac{l_{1} l_{2}}{L_{1} L_{2}} \quad \text { for a rectangular lattice; }
$$


- the structural factor for square rods, $I_{\mathrm{SQ}}(\boldsymbol{G})$, is deduced from (19) through putting $l_{1}=l_{2}=l$ :

$$
I_{\mathrm{SQ}}(\boldsymbol{G})=I_{\mathrm{RC}}(\boldsymbol{G}) \quad \text { with } \quad l_{1}=l_{2}=l .
$$

The maximum filling fraction values for rectangular lattice and rods depend on the rectangle side length ratio, $l_{1} / l_{2}$ and the lattice constant ratio, $L_{1} / L_{2}$.

\section{References}

[1] E.N. Economou, M. Sigalas, J. Acous. Soc. Am. 95, 1734 (1994).

[2] M.S. Kushwaha, P. Halevi, Appl. Phys. Lett. 64, 1085 (1994).

[3] M.S. Kushwaha, P. Halevi, L. Dobrzynski, B. Djafari-Rouhani, Phys. Rev. Lett. 71, 2022 (1993).

[4] M.S. Kushwaha, P. Halevi, G. Martinez, L. Dobrzynski, B. Djafari-Rouhani, Phys. Rev. B 49, 2313 (1994).

[5] M. Sigalas, E.N. Economou, J. Appl. Phys. 75, 2845 (1994).

[6] M. Sigalas, E.N. Economou, J. Sound Vibr. 158, 377 (1992).

[7] M. Sigalas, E.N. Economou, Solid State Commun. 86, 141 (1993).

[8] R. Martinez-Sala, J. Sancho, J.V. Sanchez-Perez, J. Llinares, F. Meseguer, Nature 378, 241 (1997).

[9] Y.Y. Chen, Zhen Ye, Phys. Rev. Lett. 87, 184301 (2001).

[10] Y.Y. Chen, Zhen Ye, Phys. Rev. E 64, 036616 (2001).

[11] D. Caballero, J. Sanchez-Dehesa, C. Rubio, R. Martinez-Sala, J.V. Sanchez-Perez, F. Meseguer, J. Llinares, Phys. Rev. E 60, R6316 (1999).

[12] D. Caballero, J. Sanchez-Dehesa, R. Martnez-Sala, C. Rubio, J.V. Sanchez-Perez, L. Sanchis, F. Meseguer, Phys. Rev. B 64, 064303 (2001).

[13] L. Sanchis, F. Cervera, J. Sanchez-Dehesa, J.V. Sanchez-Perez, C. Rubio, R. Martinez-Sala, J. Acoust. Soc. Am. 109, 2598 (2001).

[14] Z. Liu, X. Zhang, Y. Mao, Y. Zhu, Z. Yang, C.T. Chan, P. Sheng, Science 289, 1734 (2000).

[15] M. Torres, F.R. Montero de Espinosa, J.L. Aragon, Phys. Rev. Lett. 86, 4282 (2001).

[16] F. Cervera, L. Sanchis, J.V. Sanchez-Perez, R. Martinez-Sala, C. Rubio, F. Meseguer, C. Lopez, D. Caballero, J. Sanchez-Dehesa, Phys. Rev. Lett. 88, 023902 (2002).

[17] L. Sanchis, A. Hakansson, F. Cervera, J. Sanchez-Dehesa, Phys. Rev. B 67, $035422(2003)$.

[18] A. Khelif, P.A. Deymier, B. Djafari-Rouhani, J.O. Vasseur, L. Dobrzynski, J. Appl. Phys. 94, 1308 (2003).

[19] Ch.M. Anderson, K.P. Giapis, Phys. Rev. B 56, 7313 (1997).

[20] J.O. Vasseur, B. Djafari-Rouhani, L. Dobrzynski, M.S. Kushwaha, P. Halevi, J. Phys., Condens. Matter 6, 8759 (1994).

[21] R. Wang, X. Wang, B. Gu, G. Yang, J. Appl. Phys. 90, 4307 (2001). 
[22] F. Wu, Z. Liu, Y. Liu, Phys. Rev. E 66, 046628 (2002).

[23] W. Kuang, Z. Hou, Y. Liu, Phys. Lett. A 332, 481 (2004).

[24] J.O. Vasseur, B. Djafari-Rouhani, L. Dobrzynski, P.A. Deymier, J. Phys., Condens. Matter 9, 7327 (1997).

[25] L.D. Landau, E.M. Lifszyc, The Theory of Elasticity, PWN, Warszawa 1993.

[26] M. Kafesaki, M.M. Sigalas, E.N. Economou, Solid State Commun. 96, 285 (1995).

[27] J.O. Vasseur, P.A. Deymier, G. Frantziskonis, G. Hong, B. Djafari-Rouhani, L. Dobrzynski, J. Phys., Condens. Matter 10, 6051 (1998). 